\title{
Fiber Bragg grating Fabry-Perot structures under loading and their applications in switchable multi-wavelength lasers
}

\author{
Xuewen Shu, Kate Sugden \\ Photonics Research Group, Aston University, Birmingham, B4 7ET, United Kingdom \\ Email:x.shu@aston.ac.uk
}

\begin{abstract}
Characteristics of fiber Bragg grating based Fabry-Perot (FBG-FP) structures under transversal loading are investigated. A novel switchable multi-wavelength fiber laser employing loaded FBG-FP is also demonstrated.

OCIS codes: (060.3735) Fiber Bragg gratings; ( 060.3510) Lasers, fiber; (050.2230) Fabry-Perot
\end{abstract}

\section{Introduction}

Fabry-Perot (FP) resonance structures have very important applications in many areas of physics and engineering. Traditional bulk-optic or thin-film FP cavities have played a key role in many optical systems such as gas solid states lasers, semiconductor lasers, and high-precision measurement instruments. In-fiber FP structures based on fiber Bragg gratings (FBG) have also attracted considerable attention in recent years due to their important applications in optical fiber communication systems and optical fiber sensing [1-3]. FBG based FP (FBG-FP) cavities have some significant advantageous features including their full compatibility with fiber-optic systems, intrinsically stable alignment, low insertion loss and low cost. In this paper, we report the characteristics of FBG-FP cavities under transversal loading, which exhibit a very attractive and unique feature that traditional bulk-optic or thin-film FP cavities do not have. We also demonstrate here a novel switchable multi-wavelength laser employing such a loaded FBG-FP structure.

\section{Experiments}

FBG-FP structures were fabricated in B/Ge co-doped photosensitive fibers with conventional UV-laser inscription technology. The schematic of a FBG-FP under transversal loading test is shown in the inset of Fig.1a, in which a FBG-FP together with a support fiber (not shown) was sandwiched between two metal plates loaded with force. The typical transmission spectra of a FBG-FP with/without loading were shown in Fig.1a. The transmission spectra were measured with a broadband light source and an optical spectrum analyzer (OSA, Ando AQ 6317) with a
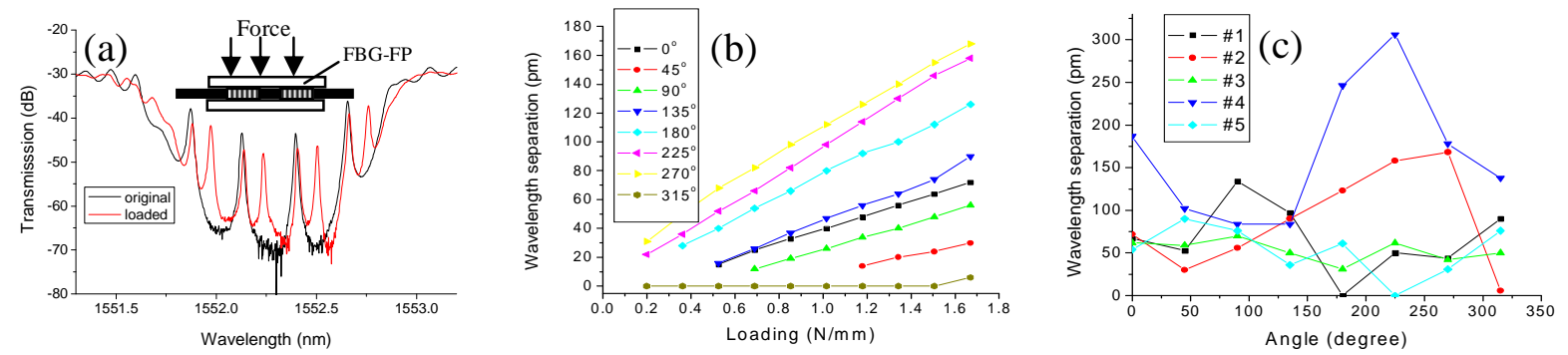

Fig.1 (a) Measured transmission spectra of a FBG-FP with/without load. Insect shows the schematic of a loaded FBG-FP. (b) Wavelength separation as a function of loading for different fiber orientations. (c) Wavelength separation as a function of fiber orientation angle for different FBG-FP samples.

resolution of $0.01 \mathrm{~nm}$. It is clearly seen in Fig.1a that the number of the sharp transmission peaks for the loaded FBG-FP was doubled compared with the original one. Each transmission peak split into a pair of peaks due to transversal load induced birefringence in the optical fiber. The two peaks for each pair belong to different polarizations, this was verified using polarized light as the input. The wavelength separation of the split peak pair was found to be almost proportional to the loading applied and was also found to be strongly dependent on the orientation of the transversal load applied. We used small, scotch tape flags attached to the fiber to indicate the orientation of the fiber. The initial $0^{\circ}$ angle was randomly chosen and the fibre was then rotated around its longitudinal axis. Fig.1b shows the response of the FBG-FP as a function of transversal loading with different fiber orientations - an effect that is dependent on how the incoming polarisation of the light aligns with the induced 
birefringence. It is seen in Fig.1b that the loading sensitivities for different fiber orientations were significantly different. At each orientation, the wavelength separation of the split peaks shows a linear relationship with the loading. We also tested different FBG-FPs of different cavity lengths. Fig.1c shows the response of five different FBG-FP samples under the same loading of $1.67 \mathrm{~N} / \mathrm{mm}$ as a function of fiber orientation angles. It is seen that different FBG-FPs had significant different responses to the transversal loading. It is also interesting to note in Fig.1c that the loading sensitivity can reach almost zero at a certain fiber orientation for some samples.

We then employed a loaded FBG-FP structure in a linear cavity fiber laser to generate multi-wavelength lasing. Fig. 2 schematically shows the experimental setup of the laser. The cavity of the multi-wavelength laser was formed by a loaded FBG-FP with an optical circulator, a polarization controller (PC), a section of Er/Yb fiber with a length of $1.2 \mathrm{~m}$ and an apodised FBG with a $3 \mathrm{~dB}$ bandwidth of $0.28 \mathrm{~nm}$. The apodised FBG was mounted on a fiber stretcher to adjust central wavelength and therefore can selectively align with transmission peaks of the loaded FBG-FP. The FBG-FP had a cavity length of $8 \mathrm{~mm}$ and a FSR of $\sim 0.12 \mathrm{~nm}$. The laser was pumped from a 980/1550 $\mathrm{nm}$ WDM coupler spliced at one end of the cavity to minimize the cavity length, and the laser output was launched into a 10:90 coupler. $10 \%$ of the laser output was monitored by the OSA, while $90 \%$ of the output was monitored

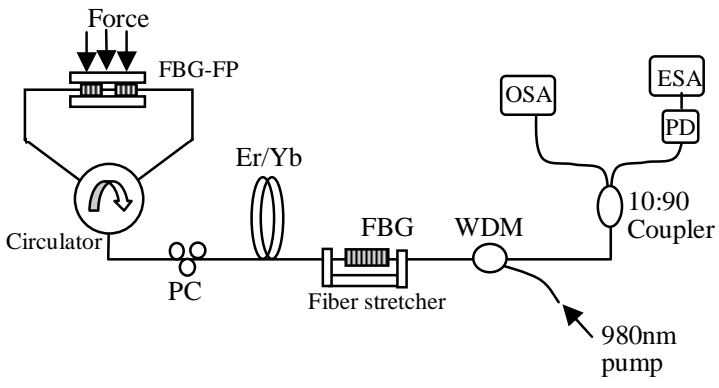

Fig.2 Schematic of the experimental setup for multiwavelength lasing.
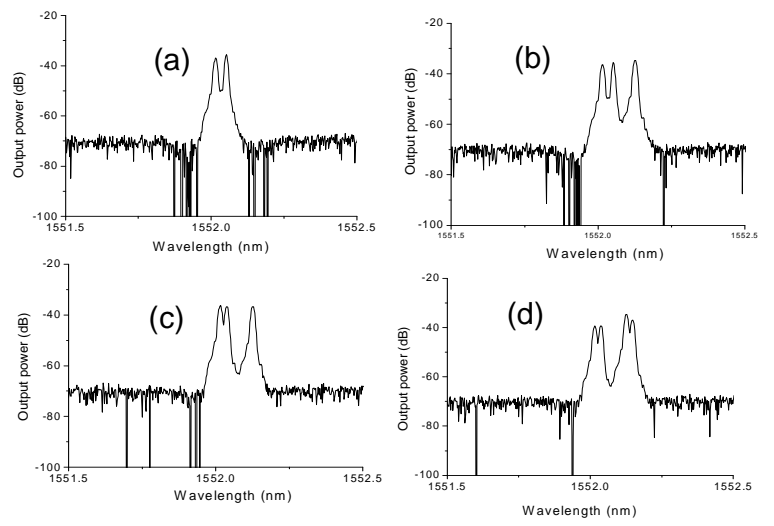

Fig.3 Examples of the measured output spectra of the multi-wavelength laser.

by an electrical spectrum analyzer (ESA). By combining adjusting the loading force, PC and fiber stretcher, the laser can be switched to operate between 1 and 4 laser wavelengths together with adjustable separation and wavelength selectability. Fig. 3 shows some typical examples of different lasing cases. Fig.3a shows a dualwavelength lasing. Fig. $3 \mathrm{~b}$ and $3 \mathrm{c}$ show triple-wavelength lasing, however the separation was different due to different transversal loading applied. Fig.3d shows a four-wavelength lasing. The proposed laser configuration here combines spatial hole-burning (due to the linear cavity) and polarization hole-burning (due to the birefringence induced by the transversal loading) effects and therefore can achieve multi-wavelength lasing at room temperature with good stability. It is should be pointed out here that the proposed laser in Fig. 2 can also be used to generate microwave signals. Through optical beating between two or more laser wavelengths, tunable single- frequency or multi-frequency microwave signals can be generated.

\section{Conclusions}

We have characterised the properties of FBG-FP structures under the transversal loading. We also proposed and demonstrated a novel switchable multi-wavelength fiber laser that employed a loaded FBG-FP structure. Loaded FBG-FP structures and their application in multi-wavelength lasers may lead to many further applications in both optical communication and optical sensing.

\section{References}

[1] G.E. Town, et al., IEEE Photon. Technol. Lett., 7 (1995), p.78.

[2] X. Shu and K. Sugden, Opt. Lett., 28 (2003), p.1897.

[3] D. Chen, et al., Electron. Lett., 44 (2008), p.459.

[4] W. Liang, et al., Appl. Lett., 86 (2005), p.151122.

[5] X. Wang and H.F.Taylor, Opt. Lett., 27 (2002), p.1388.

[6] R. Slavik, et al., J Lightwave Technol., 21 (2003) p.1059 